\title{
Detection of Outer Layer of the Vessel Wall and Characterization of Calcified Plaques in IVUS Images
}

\author{
Alireza Roodaki ${ }^{1}$, Zahra Najafi ${ }^{1}$, Armin Soltanzadi ${ }^{1}$, Arash Taki ${ }^{2}$, Seyed \\ Kamaledin Setarehdan ${ }^{1}$, Nasir $\mathrm{Navab}^{2}$ \\ 1 Control and Intelligent Processing Centre Of Excellence, Faculty of Electrical and \\ Computer Engineering, University of Tehran, Tehran, Iran \\ \{a.roodaki@ece.ut.ac.ir, ksetareh@ut.ac.ir\} \\ ${ }^{2}$ Computer Aided Medical Procedures (CAMP) - TU Munich, Germany
}

\begin{abstract}
Intravascular Ultrasound (IVUS) is a diagnostic imaging technique that provides tomographic visualization of coronary arteries. Important challenges in analysis of IVUS images are speckle noise, artifacts of catheter and calcified shadows. In this paper, we present a method for the automated detection of outer (media-adventitia) border of vessel by the use of geometric deformable models. Speckle noise is reduced with median filter. The initial contour is extracted using Canny edge detection and finally the calcified regions are characterized by using Bayes classifier and thresholding methods. The proposed methods were evaluated on 60 IVUS images from 7 different patients. The results show that the border detection method was statistically accurate and in the range of inter observer variability (based on the used validation methods). Bayesian classifier enables us to characterize the regions of interest, with a sensitivity and specificity of $92.67 \%$ and $98.5 \%$ respectively.
\end{abstract}

Keywords: Border detection, Deformable models, IVUS, Tissue characterization.

\section{Introduction}

Intravascular Ultrasound (IVUS) is a catheter-based medical imaging technique. Using a specially designed ultrasound catheter it provides real-time tomographic images of the arterial wall that shows the morphology and histological properties of the cross-section of the vessel. IVUS not only provides a quantitative assessment of the vessels' wall but also introduces information about the nature of atherosclerotic lesions as well as the plaque shape and size [1]. The first step for plaque characterization in IVUS images is detection of outer layer of vessel wall. Nevertheless, it is a difficult, subjective and time-consuming procedure to manually perform segmentation. Therefore, there is an increasing interest in developing automatic tissue segmentation algorithms for IVUS images.

Reducing the effect of the speckle noise is required for many applications in ultrasound image processing [2]. Most widely used techniques to reduce the speckle 
noise include the Median Filter. This filter seeks a balance between averaging and all pass filters, although it improves the quality of the images. Several segmentation methods in IVUS images have been proposed in the literature. Some of the earlier works on segmentation of the IVUS images were semi-automatic and some other were based on the energy minimization of a contour either guided snake toward the target structure or minimize a cost function $[5,6]$. Segmentation methods based on probabilistic approaches have been proposed in $[4,8]$. Some authors combine transversal and longitudinal contours to provide the model with spatial continuity along the sequences [7]. Region growing method is used in [9] to detect the edges.

The active contours used in the previous approaches are mostly based on a kind of parametric deformable model. However, parametric deformable models have two main limitations [10]. For recognizing calcium regions in IVUS images some characterization methods have been proposed using adaptive thresholding algorithm [3] and texture based features [13, 14]. However the thresholding based method is sharp in discriminating and ignores the variants of gray level of regions, and the latter is time consuming and doesn't have the desirable accuracy.

We have focused on the development and validation of an automated method based on non-parametric deformable models for accurate IVUS image segmentation. The results of the application of the proposed algorithm to real IVUS images of different patients are presented. After detecting the media-adventitia boundary and extracting the region between this border and the catheter, the Bayes classifier and the thresholding based method is employed to characterize the calcified regions. The calcified area detected by the implemented methods was validated and compared with the manually segmented areas by the expert.

\section{Method}

\subsection{Border Detection}

Reducing the effect of the speckle noise is required for many applications in ultrasound image processing. Median filter is used for denoising in this work [2]. The planar image in Cartesian coordinates is converted into the polar coordinates. The reason for this is that the more or less circular vessel structures can be processed easier in polar coordinates. For detecting the initial contour, a Canny edge detection method $(\alpha=8)$ is used for media-adventitia. After the border detection, the images have to be converted back to the Cartesian coordinates in order to continue edge detection with deformable model and finally to be understood well by the physicians.

Manual processing of IVUS images is a tedious and time consuming procedure. Many efforts have been made in order to develop an accurate automated method for the detection of the regions of interest in IVUS images. Many restrictions in automated segmentation of IVUS images derive from the quality of the image, such as the lack of homogeneity of regions of interest and shadowed regions, which are produced by the presence of calcium [12]. 
Let us consider a dynamic curve as $X(s, t)=[X(s, t), Y(s, t)]$ where $t$ is the time and $s$ is the curve parameter. Let us also to denote the curve's inward unit normal as $N$ and its curvature as $k$. The evolution of the curve along its normal direction can be characterized by the following partial differential equation:

$$
\frac{\partial X}{\partial t}=V(\kappa) N
$$

where $V(k)$ is the speed function since it determines the speed of the curve evolution. In the level set method, the curve is represented implicitly as a level set of a 2D scalar function which is usually defined on the same domain as the image itself. The level set is defined as the set of points that have the same function value.

We now derive the level set embedding of the curve evolution. Given a level set function $\varphi(x, y, t)$ with the contour $X(s, t)$ as its zero level set we have $\varphi[X(s, t), t]$. Differentiating this term with respect to $t$ and using the chain rule, we obtain:

$$
\frac{\partial \phi}{\partial t}+\nabla \phi \frac{\partial X}{\partial t}=0
$$

where $\nabla \varphi$ denotes the gradient of $\varphi$, assuming that $\varphi$ is negative inside the zero level set and positive outside it. Equation (2) can be rewritten to (3) according to inward unit normal to the level set curve:

$$
\frac{\partial \phi}{\partial t}=V(\kappa)|\nabla \phi|
$$

where $k$ the curvature at the zero level set is given by:

Since the evolution (3) is derived for the zero level set only, the speed function $V(k)$, in general, is not defined on other level sets. Hence, we need a method to extend the speed function $V(k)$ to all of the level sets. A speed function that is used by geometric deformable contours, takes the following form:

$$
\frac{\partial \phi}{\partial t}=c\left(\kappa+V_{0}\right)|\nabla \phi|
$$

where

$$
c=\frac{1}{1+\left|\nabla\left(G_{\sigma} * I\right)\right|}
$$

A positive value of $V_{0}$ shrinks the curve while a negative $V_{0}$ expands it. The curve evolution is coupled with the image data through a multiplicative stopping term. This scheme can work well for objects that have good contrast. However, when the object boundary is indistinct or has gaps like the IVUS image in our case, the geometric deformable contour may leak out because the multiplicative term only slows down the curve near the boundary rather than completely stopping the curve. Once the curve passes the boundary, it will not be pulled back to recover the correct boundary.

To overcome this deficiency a new term is added to (5) as shown in (7).

$$
\frac{\partial \phi}{\partial t}=c\left(\kappa+V_{0}\right)|\nabla \phi|+\nabla c \nabla \phi
$$

The resulting speed function has an extra stopping term $\nabla C \nabla \varphi$ that can pull back the contour if it passes the boundary [11]. 


\subsection{Characterizing Calcified Region}

In the previous section, media-adventitia boundary was detected with geometric deformable model method. The region between this border and the catheter is extracted. Vessels' plaques are generally composed of calcium, fibrous and lipid. Calcified regions in IVUS images can be recognized by following characteristics:

- They are usually represented by bright intensity among plaque region.

- As calcium is a hard plaque, the ultrasound beam is not strong enough to penetrate through it. Therefore, the calcified region is usually followed by a dense shadow. Two methods for characterizing calcified regions are examined here. The first one is Bayes classifier and the other is implemented via setting a threshold value on the pixel intensities. For both techniques the results are confirmed by checking the shadow behind the calcified region.

\subsubsection{Bayes Classifier}

Bayes classifier is based on maximum a posteriori probability, in which the feature vector $X$ is assigned to class $\omega_{\mathrm{j}}$ if

$$
\mathrm{P}\left(\omega_{\mathrm{j}} \mid \mathrm{X}\right) \succ \mathrm{P}\left(\omega_{\mathrm{k}} \mid \mathrm{X}\right), k=1,2 \ldots M,
$$

where $M$ is the number of classes and $P$ is the probability. As the amount of a posteriori probabilities are unknown in most application, its quantity should be calculated through Bayes algorithm where

$$
P(\omega \mid X)=\frac{P(X \mid \omega) \times P(\omega)}{P(X)}
$$

From the above equation and considering that $P(X)$ is equal for all classes, (6) can be written as

$$
P\left(X \mid \omega_{j}\right) \times P\left(\omega_{j}\right) \succ P\left(X \mid \omega_{k}\right) \times P\left(\omega_{k}\right), k=1,2 \ldots M
$$

Here we have two classes, one calcified $\left(\omega_{1}\right)$ and another non-calcified $\left(\omega_{2}\right)$. The Probability Density Function (pdf) of classes is assumed to be Gaussian; therefore, only the values of mean and variance of pdf's should be estimated. These values are attained from 30 images, which were collected by the expert from the dataset, and set as follows: $\mathrm{mu}_{1}=245, \mathrm{mu}_{2}=70, \operatorname{sig}_{1}=20, \operatorname{sig}_{2}=20$.

The values of a priori probabilities are achieved experimentally from 30 images and are as follows: $\mathrm{P}($ calcified region $)=0.1, \mathrm{p}($ Non-calcified region $)=0.9$.

\subsubsection{Thresholding Method}

In this method, by studying the pixels' intensities for two classes (calcified and noncalcified) in 30 images, the average value is achieved and is set to 173.50 . Therefore, the pixel intensities which are greater than the threshold value are set to calcified class and the others are set to non-calcified class. 


\subsubsection{Checking the Shadows}

In order to increase our classifier reliability, the shadows following the detected calcified regions should get verified and in the case of existing shadow, these identified regions are accepted to be calcified region, otherwise they are set back to non-calcified class. As the IVUS images have circular trait, we first transform the images to polar coordinates where the shadows behind detected calcified regions can easily be checked. For this goal, average of pixels' intensities, which are placed in the same angle as identified regions, is calculated and the one that it's value is below 70 , is accepted to remain as calcified class.

\section{$3 \quad$ Result and Discussion}

Our study group as sequences of IVUS images includes 7 patients. These images with the digitized matrix size of $500 * 500$ pixels were acquired using a $30-\mathrm{MHz}$ transducer at a $0.5 \mathrm{~mm} / \mathrm{s}$ pullback speed. 60 frames from each patient have been gathered. The accuracy of the mentioned method is determined by comparing this method resulting borders with borders identified by an expert.

\subsection{Border Detection}

To put the accuracy of the segmentation into numbers, average distance (AD) and Hausdorff distance, the maximum distance between boundaries, (HD) between automatic and manually traced borders were calculated. These distances directly depict point to point contour variations. The level set based method was applied to each frame to detect intimia and medi-adventitia layers by defining a distance function from the initial border that were detected in preprocessing step. Fig. 2 shows an example outcome of the method.
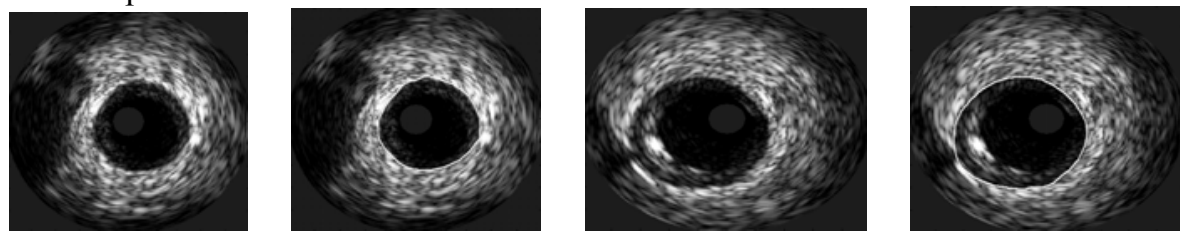

Fig. 2. Normal and calcified original images (number 1 and 3) and the result of method (number 2 and 4).

The cross sectional media_adventitia distances from manually traced and automatically detected boundaries were compared, and the results were expressed as systematic and random differences (Means \pm Standard deviation). The automatically determined borders corresponded very well with expert manual measurements. The Average (AD) and Hausdorff distance (HD) values were obtained for the proposed method, demonstrating that this method is powerful for simulated IVUS segmentation (because of the low value for these measurements). These two values for the first expert were $0.344 \pm 0.108$ and $0.650 \pm 0.150(\mathrm{~mm})$ respectively. For the second expert 
the values for $\mathrm{AD}$ and $\mathrm{HD}$ were $0.360 \pm 0.100$ and $0.780 \pm 0.180(\mathrm{~mm})$. Area difference value was calculated for the non-overlapping area borders of proposed method and parametric deformable model, the result was $12.3 \pm 1.9$ and $13.3 \pm 2.4\left(\mathrm{~mm}^{2}\right)$ respectively.

\subsection{Classification Accuracy}

The calcified regions detected by the implemented methods were also compared with the manually detected images. In order to validate the system, the sensitivity and specificity of calcified class were calculated. For this purpose true and false positive (TP and FP), and true and false negative (TN and FN) values were computed and sensitivity and specificities were calculated. The sensitivity for a class is the percentage of members of that class that are correctly classified by the test. As such, it has to be as high as possible. The specificity for a class is the percentage of members of the other classes that are correctly classified by the test. Table 1 shows these values for two classification method. The results of different steps of characterizing calcified region by means of Bayes classifier are demonstrated in Fig. 3.

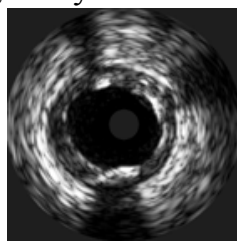

(a)

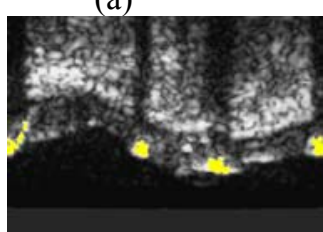

(d)

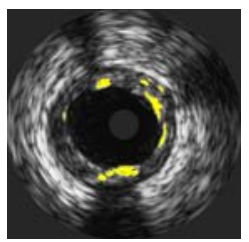

(b)

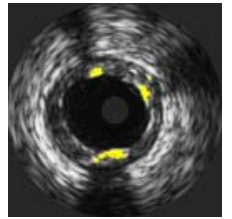

(e)

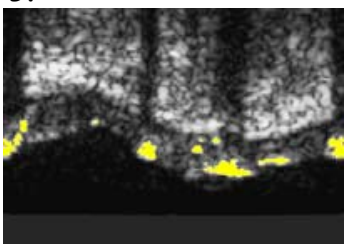

(c)

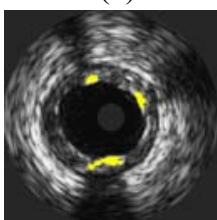

(f)

Fig. 3. Characterizing calcified region. (a) IVUS image, (b) High intensity plaque identification by Bayes algorithm ( yellow regions indicates calcified area), (c) Transformed image in Polar coordinate, (d) Removing identified plaques which are not followed by shadow, (e) Reconstructed image in Cartesian coordinate ( final result), (f) Manually characterized calcified image by expert.

Table 1. Performance of Calcified region characterization methods.

\begin{tabular}{|c|c|c|}
\hline Classification Method & Sensitivity & Specificity \\
\hline Bayes Classifier & $92.674 \%$ & $\mathbf{9 8 . 5 \%}$ \\
\hline Thresholding method & $74.14 \%$ & $\mathbf{8 3 . 7 \%}$ \\
\hline Adaptive Thresholding [6] & $\mathbf{8 4 \%}$ & $\mathbf{8 8 \%}$ \\
\hline Texturural Features [14] & $76.04 \%$ & Not-reported \\
\hline
\end{tabular}




\section{Conclusion}

Border detection and region identification in IVUS images are a challenging task in medical imaging analysis. Few algorithms have been developed in order to trace media-adventitia border automatically. In this paper the preprocessing includes median filtering that reduces the noise well and preserves the edges of the image and second, detection of the initialize contour with edge detection methods that makes the deformable model method automatic. The initial contour is defined to be a distance function for the evolution equation. After detecting the border, calcified regions are identified with Bayesian classifier and thresholding method. In our validation methodology we compared the results from the implemented methods with the manual estimation of borders by an expert. We observed small variations between manual and automated detection of borders, this denotes that this automatic method was accurate.

Geometric deformable models have some advantages over parametric models. First, they are completely intrinsic and, therefore, are independent of the parameterization of the evolving contour. In fact, the model is generally not parameterized until evolution of the level set function is complete. Second, the intrinsic geometric properties of the contour, such as the unit normal vector and the curvature, can be easily computed from the level set function. We have solved the problem of the places of the nodes in initialization stage for both methods. The proposed method has limitations such that it is not accurate where there is the artifact of existence of other branches or the artifact of curvature of the vessel or catheter and sometimes the frames with calcified shadowing artifacts, for this reason we suggest to use other frames around this frame for decision of the place of the borders.

Also in this paper, the ability of Bayes classifier in characterizing calcified region was investigated. The results of our study show that this method has improved the value of sensitivity and specificity in comparison with other algorithms such as thresholding or using texture based features.

Acknowledgments. The authors would like to thank Mr. Shaki for his suggestions and valuable comments and VOLCANO Company for their help in providing the data set.

\section{References}

1. Schoenhagen, P., Stillman, A. E.: Principles, Advances, Clinical Uses of IVUS. Clinic journal of medicine, 72, 43--45 (2005)

2. Michailovich, O. V., Tannenbaum, A.: Despeckling of Medical Ultrasound Images. IEEE Trans. on Ultrasonics, ferroelectrics and frequency control, 53, 64--78 (2006)

3. Filho, E. D., Yoshizawa, M.: A Study on Intravascular Ultrasound Image Processing. Journal of Mathematical Imaging and Vision, 21, 205--223 (2004)

4. Gil, D., Radeva, P., Saludes, J.: Segmentation of Artery Wall in Coronary IVUS Images: A Probabilistic Approach. Computer in Cardiology, 687--690 (2000) 
5. Bourantas, V., Plissiti, E., Fotiadis, I., Protopappas, C., Mpozios, V., Katsouras, S., Kourtis C., Rees, M. R., Mivhalis, K.: In vivo Validation of a Novel Semi-automated Method for Border Detection in Intravascular Ultrasound Images. The British Journal of Radiology, 78, 122--129 (2005)

6. Wang, L. Y., Wang, W.: Estimating Coronary Artery Lumen Area with Optimizationbased Contour Detection. IEEE Trans. Med. Imag., 22, 48--6 (2003)

7. Papadogiorgaki, M., Mezaris, V., Chatzizisis, Y. S., Kompatsiaris, I., Giannoglou, G. D.: A Fully Automated Texture-based Approach for the Segmentation of Sequential IVUS Images. International Conference on Systems, Signals \& Image Processing, 8, 461--464 (2006)

8. Cardinal, M., Meunier, J., Soulez, G., Maurice, R. L.: Intravascular Ultrasound Image Segmentation: A Three-dimensional Fast-marching Method Based on Gray Level Distributions. IEEE Trans. Med. Imag., 25, 590--601 (2006)

9. Brathwaite, P. A., Chandran, K. B, McPherson, D. D., Dove, E. L.: Lumen Detection in Human IVUS Images Using Region-growing. IEEE Conference in Cardiology, 8, 37-40 (1996)

10. McInerney, T., Terzopoulos, D.: Deformable Models in Medical Image Analysis: A Survey. Med. Imag. Anal., 1, 91--108 (1996)

11. Han, X., Xu, C., Joil, P.: A Topology Preserving Level Set Method for Geometric Deformable Models. IEEE Computer Society, 25, 755--768 (2003)

12. Kass, M., Witkin, A., Terzopoulos, D.: Snakes: Active Contour Models. Int. J. Comp. Vision, 1, 321--331 (1987)

13. Vince, D. G., Dixon, K. J., Cothren, R. M., Cornhill, J. F.: Comparison of Texture Analysis Methods for the Characterization of Coronary Plaques in Intravascular Ultrasound Images. Computerized Medical Imaging and Graphics, 24, 221--229 (2000)

14. Caballero, K. L., Barajas J., Pujol, O., Mauri, J., Radeva, P.: In-Vivo IVUS Tissue Classification: A Comparison between RF Signal Analysis and Reconstructed Images. CIARP, 137--146 (2006) 